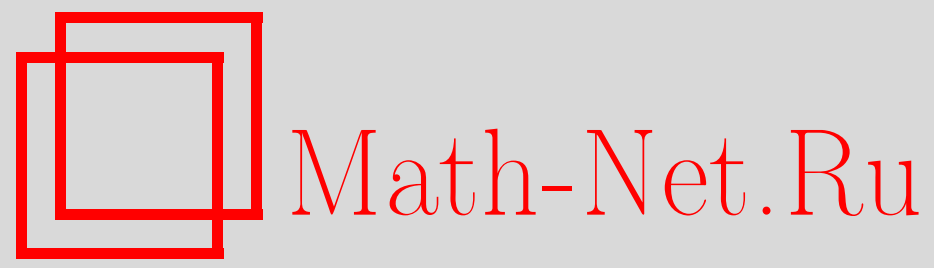

П. В. Ролдугин, Редукция графа при поиске реберного покрытия минимальным числом клик, Матем. вопр. криптогр., 2012, том 3, выпуск 3, 105-128

DOI: https://doi.org/10.4213/mvk63

Использование Общероссийского математического портала Math-Net.Ru подразумевает, что вы прочитали и согласны с пользовательским соглашением

http://www . mathnet.ru/rus/agreement

Параметры загрузки:

IP : 54.92 .164 .108

26 апреля 2023 г., 13:41:32 
УДК: 519.177.3+519.178

\title{
Редукция графа при поиске реберного покрытия Минимальным Числом КЛИк
}

\author{
П. В. Ролдугин \\ Московский государственный технический университет радиотехники, \\ электроники и автоматики, Москва
}

Получено 20.V.2011

В статье рассматривается задача построения покрытия всех ребер графа минимальным числом его полных подграфов (клик). Описаны классы подграфов, позволяющих сводить задачу поиска указанного покрытия к такой же задаче для графов меньшего порядка. Показано, что эти классы подграфов являются более общими по отношению к классам, предлагавшимся ранее.

Ключевые слова: классы графов, покрытия графов, клики

Graph reduction in the construction of minimal clique cover

\section{P. V. Roldugin}

Moscow State Technical University of Radio Engineering, Electronics and Automatics, Moscow

Abstract. The problem of construction of a graph coverage with a minimum number of its complete subgraphs (cliques) is considered. We describe classes of subgraphs for which it is possible to reduce the graph coverage problem to the same problem for graphs of smaller orders. We show that these classes are wider than those known before.

Key words: classes of graphs, graph coverings, cliques

Citation: Mathematical Aspects of Cryptography, 2012, vol. 3, no. 3, pp. 105-128 (Russian). 


\section{Введение}

Реберным покрытием графа называется такой набор его подграфов, что любое ребро графа лежит в одном из подграфов набора. Если все подграфы в наборе являются кликами, т. е. полными подграфами, то говорят о реберном покрытии графа кликами (РПК). Известной алгоритмической задачей в теории графов является построение РПК из минимального числа клик. Данная задача является $N P$-полной (более строго: задача определения по графу $G$ и числу $r$ наличия РПК из $r$ клик является $N P$-полной, см. [7]), поэтому основные усилия исследователей в этой области сосредоточены на построении алгоритмов поиска РПК, позволяющих снизить трудоемкость полного перебора.

В криптографии задача поиска РПК минимальным числом клик возникла при рассмотрении так называемого «конфликта ключей» («keyword conflict»), впервые в зарубежной печати описанного в работе Е. Келлермана (Kellerman E., [1]). Суть указанной проблемы в одной из интерпретаций можно описать следующим образом. В сети связи с конечным числом абонентов между некоторыми парами абонентов установлены доверительные отношения (например, имеются общие вычислительные ресурсы), а между некоторыми - нет. На всю сеть имеется набор из $r$ ключей; у каждого из абонентов имеется какой-то поднабор из этого набора. Эти поднаборы должны обладать двумя «естественными» свойствами: если два абонента доверяют друг другу, то в их поднаборах найдется общий ключ; если между парой абонентов нет доверия, то и общего ключа у них быть не должно. В [1] показано, что задача нахождения минимального $r$, при котором возможно построение набора с указанными свойствами, эквивалентна задаче нахождения минимального числа клик, покрывающих все ребра графа.

Введем строгое определение. Реберным покрытием кликами (РПК) графа $G=(V, E)$ называется такой набор клик (полных подграфов) $K_{1}, \ldots, K_{r}$, что любое ребро и любая вершина графа $G$ лежат хотя бы в одной из клик $K_{1}, \ldots, K_{r}$. В РПК $K_{1}, \ldots, K_{r}$ каждую из клик можно дополнить до максимальной клики (максимальной по включению) и получить также РПК из того же числа клик; поэтому считаем, что в определении РПК речь идет о максимальных кликах. Минимально возможное число клик, которым может быть покрыт граф $G$, называется кликовым числом графа $G$ и обозначается через $\Theta(G)$; РПК из $\Theta(G)$ клик называется минимальным РПК. Из литературы, относящейся к проблеме построения минимального РПК, можно указать работы [1-6]. В частности, доказано, что задача нахождения $\Theta(G)$ и минимального РПК является полиномиальной, если максимальная степень вершин графа не больше 5. Для графов с максимальной степенью 6 задача уже $N P$-полна. 
Используем следующие определения и обозначения. Порожденным подграфом $H$ в графе $G$ называется подграф $H$, для которого выполнено условие: если $x, y \in V(H)$ и $(x, y) \in E(G)$, то $(x, y) \in E(H)$. Говорят также, что подграф $H$ порожден множеством вершин $V(H)$. Если $H$ - подграф или множество вершин графа $G$, то через $G \backslash H$ обозначим граф, полученный из $G$ удалением всех вершин $H$ и инцидентных им в графе $G$ ребер; если $H$ - подграф графа $G$, то обозначим через $G \backslash E(H)$ граф $(V(G), E(G) \backslash E(H))$. Через $N_{G}(x)$ обозначим окружение вершины в графе $G$, т. е. $N_{G}(x)=\{y \in V(G):(x, y) \in E(G)\}$. Будем писать просто $N(x)$, когда ясно, о каком графе идет речь. Через $N^{*}(x)$ обозначим замкнутое окружение вершины $x$, т. е. $N^{*}(x)=N(x) \cup\{x\}$. Кроме того, для подграфов, порожденных множествами вершин $N(x)$ и $N^{*}(x)$, будем использовать те же обозначения и названия. Подмножество $U$ множества ребер графа $G$ называется ребернымм покрытием вершин, если каждая вершина графа $G$ инцидентна хотя бы одному ребру из $U$ (отметим, что в этом определении, в отличие от определения РПК, речь идет о покрытии множества вершин). Для простоты полный подграф порядка 3 иногда будем называть треугольником; термины теории графов, не определяемые, но используемые в статье, можно найти в [9].

Задача поиска минимального РПК решается весьма трудоемким перебором; фактически в настоящее время основным путем снижения трудоемкости является нахождение в графе объектов (вершин, ребер, подграфов с особыми свойствами и так далее), которые можно «изьять»из графа с тем, чтобы найти в редуцированном графе минимальное РПК и затем с малой трудоемкостью построить минимальное РПК для исходного графа. Именно такой подход, в частности, использован в работах $[3,5]$.

Примером редукции графа в задаче поиска минимального РПК графа $G$ может служить случай, когда граф $G$ не связен. Тогда можно найти минимальное РПК для каждой из компонент связности: их объединение и будет минимальным РПК всего графа $G$. Отметим, что понятие редукции формально не определяется, поскольку это и не требуется; нестрого говоря, под понятием редукции графа понимаются утверждения, позволяющие сводить задачу поиска минимального РПК к такой же задаче на одном или нескольких подграфах исходного графа. Разумеется, построение минимального РПК для исходного графа по минимальному РПК для редуцированного графа должно иметь небольшую трудоемкость, по возможности линейную по числу вершин или числу ребер.

В настоящей статье рассмотрены такие подграфы, как выделенный подграф и компакт, и доказано, что эти подграфы позволяют проводить редукцию графа в задаче поиска минимального РПК. Показано, что частными случаями 
выделенного подграфа и компакта являются все объекты, введенные в работах $[3,5]$. Установлена полиномиальная трудоемкость поиска рассмотренных подграфов. Кроме того, для подграфов, известных ранее, рассмотрен ряд важных для задачи поиска минимального РПК свойств.

\section{§1. Собственные ребра подграфа и выделенные подграфы}

Центральным для настоящей работы является следующее понятие. Собственным ребром порожденного подграфа $H$ графа $G$ назовем такое ребро $e=(x, y)$ подграфа $H$, что если ребро $e$ лежит в треугольнике $\{x, y, z\}$ графа $G$, то вершина $z$ принадлежит множеству вершин подграфа $H$.

Для собственных ребер верна следующая лемма, напрямую вытекающая из определения.

Лемма 1. Если порожденный подграф $H$ графа $G$ содержит собственное ребро е, то в любом РПК графа $G$ все клики, содержащие ребро е, иеликом лежат в подграфе $H$.

Доказательство. Пусть в некотором РПК графа $G$ есть клика $K$, содержащая собственное ребро $e=(x, y)$, которая не лежит целиком в $H$. Тогда существует вершина $z$ в клике $K$, которая не принадлежит множеству вершин подграфа $H$. Следовательно, $z$ отлична от $x$ и $y$, и, поскольку $K-$ клика, вершина $z$ смежна и с $x$, и с $y$. Таким образом, $\{x, y, z\}-$ треугольник, существование которого противоречит определению собственного ребра.

Эта лемма приводит нас к следующему объекту. Очевидно, что $\Theta(G) \leq$ $\leq \Theta(G \backslash E(H))+\Theta(H)$, поскольку любое РПК подграфа $H$, объединенное с произвольным РПК подграфа $G \backslash E(H)$, дает РПК всего графа $G$. Однако указанное неравенство, вообще говоря, не всегда является равенством; один из случаев, когда это выполняется, описывается следующим утверждением. Назовем порожденный подграф $H$ графа $G$ выделенным, если все ребра этого подграфа собственные.

Утверждение 1. Пусть $G$ - граф и $H$ - его выделенный подграф. Тогда все клики из любого РПК графа $G$ можно разбить на две группы: клики, образуюшие РПК графа $H$, и клики, образующие РПК графа $G \backslash E(H)$.

Доказательство. По лемме 1 каждая из клик произвольного РПК графа $G$, которая содержит хотя бы одно ребро подграфа $H$, целиком лежит в $H$. Взяв все клики из РПК, покрывающие граф $H$, получаем, что они все лежат в $H$, т. е. образуют его РПК. Остальные клики, очевидно, образуют РПК графа $G \backslash E(H)$. 
Следствие 1. В условиях утверждения 1 справедливо равенство $\Theta(G)=\Theta(G \backslash E(H))+\Theta(H)$.

Доказательство. Возьмем минимальное РПК графа $G$; оно состоит из $\Theta(G)$ клик. Согласно утверждению 1, это РПК раскладывается на две группы клик: одна образует РПК графа $H$ (и состоит из $s$ клик); вторая образует РПК графа $G \backslash E(H)$ (и состоит из $\Theta(G)-s$ клик). Но тогда $s \geq \Theta(H)$ и $\Theta(G)-s \geq \Theta(G \backslash E(H))$. Следовательно,

$$
\Theta(G) \geq \Theta(G \backslash E(H))+s \geq \Theta(G \backslash E(H))+\Theta(H),
$$

и, значит, $\Theta(G)=\Theta(G \backslash E(H))+\Theta(H)$.

Из утверждения 1 и следствия 1 очевидно получаем следующее.

Следствие 2. В условиях утверждения 1 минимальное РПК графа $G$ состоит из двух непересекаюшихся частей: клик, образующих минимальное РПК графа $H$, и клик, образующих минимальное РПК графа $G \backslash E(H)$.

Отметим, что условиям утверждения 1 в качестве $H$ удовлетворяет любое независимое множество вершин, т. е. порожденный подграф, не содержащий ребер вообще. Однако для такого графа $\Theta(H)=0$ и $G \backslash E(H)=G$, т. е. изменения задачи поиска минимального РПК не происходит. Аналогичным вырожденным случаем является подграф $H$, равный всему графу $G$. Такие выделенные подграфы назовем тривиальными.

Собственно редукцией в задаче поиска минимального РПК графа $G$ в случае существования нетривиального выделенного подграфа $H$ будет переход к поиску минимального РПК в двух независимых графах: $H$ и $G \backslash E(H)$. Каждый из этих графов меньше графа $G$ хотя бы на одно ребро. Однако надо отметить, что если число вершин в выделенном подграфе $H$ либо мало, либо близко к числу вершин $G$, то один из графов $H$ и $G \backslash E(H)$ будет не намного меньше, чем собственно исходный граф $G$, что означает небольшой выигрыш в трудоемкости построения минимального РПК.

Заметим также, что свойство «быть выделенным подграфом» является наследственным. Более строго, верна лемма.

Лемма 2. Пусть $H$ - выделенный подграф в графе $G$ и $F-$ выделенный подграф в графе $H$. Тогда $F$ - выделенный подграф в графе $G$.

Доказательство. Пусть в подграфе $F$ найдется такое ребро $(a, b)$, что существует треугольник $\{a, b, c\}$ в графе $G$, где $c$ - вершина, не лежащая в подграфе $F$. Тогда, если $c$ лежит в $H$, то это противоречит тому, что $F-$ выделенный подграф в $H$; если $c$ не лежит в $H$, то получаем треугольник $\{a, b, c\}$, где $(a, b)$ - ребро $F$ (и, следовательно, ребро $H$ ), т. е. противоречие с тем, что $H-$ выделенный подграф в $G$. 
Лемма 2 показывает, что выделенные подграфы являются «одноразовыми», т. е. после редукции путем, указанным в утверждении 1, новых подграфов, содержащих только собственные ребра, в графах $H$ и $G \backslash E(H)$ не образуется. Это значит, что после редукции для дальнейшего решения задачи придется пользоваться другими способами нахождения минимального РПК.

Рассмотрим вопросы о критерии условия «быть выделенным подграфом» и о трудоемкости поиска таких подграфов. Для произвольного графа $G$ определим граф $\Delta_{G}$ следующим образом: $V\left(\Delta_{G}\right)=E(G)$ и $\left(e_{1}, e_{2}\right) \in E\left(\Delta_{G}\right)$ тогда и только тогда, когда в графе $G$ существует треугольник, двумя сторонами которого являются ребра $e_{1}$ и $e_{2}$ (т. е. $e_{1}=(a, b), e_{2}=(b, c)$ и $\{a, b, c\}-$ треугольник в графе $G)$.

Чтобы получить описание всех выделенных подграфов графа $G$, потребуется несколько вспомогательных лемм.

Лемма 3. Пусть $e-$-ребро графа $G$, лежащее в некотором выделенном подграфе $H$, , $f$ - ребро графа $G$, не лежамее в подграфе $H$. Тогда в графе $\Delta_{G}$ отсутствует ребро $(e, f)$.

Доказательство. Предположим противное: вершины $e$ и $f$ графа $\Delta_{G}$ смежны. По определению это значит, что $e=(a, b), f=(b, c)$ и $\{a, b, c\}-$ треугольник в графе $G$. По условию ребро $e=(a, b)$ лежит в $H$ : это подразумевает, что вершины $a$ и $b$ принадлежат подграфу $H$. Но ребро $f=(b, c)$ не лежит в $H$, и, поскольку $H$ - порожденный подграф, вершина $c$ не принадлежит $H$. Значит, в треугольнике $\{a, b, c\}$ одна сторона $(a, b)$ есть ребро $H$, а третья вершина не лежит в $H$. Это противоречит тому, что $H-$ выделенный подграф.

Лемма 4. Если в графе $G$ выделенный подграф $H$ имеет ребро $е$ и в графе $\Delta_{G}$ вершина е смежна с вершиной $f$, то ребро $f$ лежит в подграфе $H$.

Доказательство. Если $e=(a, b), f=(b, c),\{a, b, c\}-$ треугольник в графе $G$ и ребро $f$ не лежит в $H$, то, поскольку $H$ - порожденный подграф, вершина $c$ не лежит в подграфе $H$. Следовательно, имеем противоречие с определением выделенного подграфа.

Из доказанных лемм очевидно вытекает следующее утверждение.

Утверждение 2. Если $H$ - выделенный подграф графа $G$, то

$$
E(H)=V\left(W_{1}\right) \cup V\left(W_{2}\right) \cup \ldots \cup V\left(W_{s}\right),
$$

где $W_{1}, \ldots, W_{s}$ есть некоторые компоненты связности графа $\Delta_{G}$. 
Для полного описания выделенных подграфов теперь необходимо указать, каким свойством должна обладать совокупность компонент связности графа $\Delta_{G}$, чтобы объединение множеств вершин компонент из этой совокупности образовывало множество ребер выделенного подграфа.

Пусть $W_{1}, \ldots, W_{S}$ - компоненты связности графа $\Delta_{G}$ (это не обязательно все компоненты связности графа $\left.\Delta_{G}\right)$. Множество вершин этих компонент $V\left(W_{1}\right) \cup V\left(W_{2}\right) \cup \ldots \cup V\left(W_{s}\right)$ есть некоторое множество ребер в графе $G$. Рассмотрим $M\left(W_{1}, \ldots, W_{s}\right)-$ множество тех вершин графа $G$, которые инцидентны хотя бы одному ребру из $V\left(W_{1}\right) \cup V\left(W_{2}\right) \cup \ldots \cup V\left(W_{s}\right)$. Тогда ребра из $V\left(W_{1}\right) \cup V\left(W_{2}\right) \cup \ldots \cup V\left(W_{s}\right)$ соединяют вершины из множества $M\left(W_{1}, \ldots, W_{s}\right)$. Совокупность $W_{1}, \ldots, W_{s}$ назовем замкнутой, если подграф $H$ графа $G$ с $V(H)=M\left(W_{1}, \ldots, W_{s}\right)$ и $E(H)=V\left(W_{1}\right) \cup V\left(W_{2}\right) \cup$ $\cup \ldots \cup V\left(W_{s}\right)$ является порожденным подграфом. Иначе говоря, для любой пары различных вершин $a, b \in M\left(W_{1}, \ldots, W_{s}\right)$ ребро $(a, b)$, рассматриваемое как вершина графа $\Delta_{G}$, лежит в одной из компонент связности $W_{1}, \ldots, W_{s}$.

Утверждение 3. Если $H$ - выделенный подграф графа $G$, то множество его ребер совпадает с множеством вериин некоторой замкнутой совокупности компонент связности графа $\Delta_{G}$. $C$ другой стороны, если $W_{1}, \ldots, W_{S}$ есть замкнутая совокупность компонент связности графа $\Delta_{G}$, то множество вершин $V\left(W_{1}\right) \cup V\left(W_{2}\right) \cup \ldots \cup V\left(W_{s}\right)$ этих компонент совпадает с множеством ребер $E(H)$ некоторого выделенного подграфа $H$ графа $G$.

Доказательство. Сначала докажем первую часть утверждения. По утверждению 2 имеем $E(H)=V\left(W_{1}\right) \cup V\left(W_{2}\right) \cup \ldots \cup V\left(W_{s}\right)$, где $W_{1}, \ldots, W_{s}$ есть совокупность компонент связности графа $\Delta_{G}$. Остается доказать, что эта совокупность замкнутая, т. е. что для любой пары вершин $a, b$, каждая из которых инцидентна хотя бы одному ребру из $V\left(W_{1}\right) \cup V\left(W_{2}\right) \cup \ldots \cup V\left(W_{s}\right)$, ребро $(a, b)$, рассматриваемое как вершина графа $\Delta_{G}$, лежит в одной из компонент связности $W_{1}, \ldots, W_{s}$. Но, поскольку $E(H)=V\left(W_{1}\right) \cup V\left(W_{2}\right) \cup \ldots \cup$ $\cup V\left(W_{s}\right)$, вершины $a, b$ инцидентны каким-то ребрам из $E(H)$ тогда и только тогда, когда $a$ и $b$ являются вершинами подграфа $H$. Так как $H-$ порожденный подграф, то ребро $(a, b)$ обязано лежать в $E(H):(a, b) \in E(H)=$ $=V\left(W_{1}\right) \cup V\left(W_{2}\right) \cup \ldots \cup V\left(W_{s}\right)$, т. е. вершина $(a, b)$ графа $\Delta_{G}$ лежит в одной из компонент связности $W_{1}, \ldots, W_{s}$.

Докажем вторую часть утверждения. Пусть $W_{1}, \ldots, W_{s}-$ замкнутая совокупность компонент связности графа $\Delta_{G}$. Рассмотрим порожденный подграф $H$ графа $G$ с множеством вершин $M\left(W_{1}, \ldots, W_{s}\right)$. По определению замкнутой совокупности множество ребер подграфа $H$ совпадает c $V\left(W_{1}\right) \cup V\left(W_{2}\right) \cup \ldots \cup V\left(W_{s}\right)$. 
Докажем, что $H$ - выделенный подграф. Если $H-$ не выделенный подграф, то существует такой треугольник $\{a, b, c\}$ в графе $G$, что ребро $(a, b) \in E(H)$ и $c \notin V(H)$. Но тогда ребра $(a, c)$ и $(b, c)$, рассматриваемые как вершины графа $\Delta_{G}$, смежны с $(a, b)$. Так как $V(H)=M\left(W_{1}, \ldots, W_{s}\right)$, то $a, b \in M\left(W_{1}, \ldots, W_{s}\right)$, и по определению замкнутой совокупности ребро $(a, b)$ графа $\Delta_{G}$ есть вершина одной из компонент связности $W_{1}, \ldots, W_{s}$. Но тогда ребра $(a, c)$ и $(b, c)$ обязаны лежать в той же компоненте связности и, значит, $c \in M\left(W_{1}, \ldots, W_{s}\right)=V(H)$. Это противоречит тому, что $c \notin V(H)$. Следовательно, $H$ - выделенный подграф.

Таким образом, согласно доказанному утверждению нахождение всех выделенных подграфов графа $G$ эквивалентно нахождению всех замкнутых совокупностей компонент связности графа $\Delta_{G}$. Пользуясь утверждением 3 , построим алгоритм проверки существования в графе $G$ нетривиального выделенного подграфа (и нахождения такого подграфа).

Будем считать, что граф $G$ порядка $n$ хранится в оперативной памяти в таком виде, что проверить наличие в графе ребра между двумя фиксированными вершинами можно за одну элементарную операцию (например, граф хранится в виде матрицы смежности и существование ребра устанавливается за одно обращение в память). Тогда для двух ребер $e$ и $f$ графа $G$ установить их смежность в графе $\Delta_{G}$ можно также за одно обращение в память: надо убедиться, что у ребер $e$ и $f$ есть общая вершина и что две другие вершины, инцидентные $e$ и $f$, смежны в графе $G$. Таким образом, установить, являются ли две вершины графа $\Delta_{G}$ смежными, можно за фиксированное число элементарных операций.

Известно, что в случае представления графа порядка $n$ матрицей смежности трудоемкость поиска компонент связности оценивается величиной $C \cdot n^{2}$, где $C-$ константа. Значит, получить все компоненты связности графа $\Delta_{G}$ можно с трудоемкостью не более $C \cdot n^{4}$. Отметим, что для хранения вершин всех компонент связности графа $\Delta_{G}$ потребуется память не более $C^{\prime} \cdot n^{4}$.

Предположим, что в графе $G$ существует нетривиальный выделенный подграф $H$. Пусть $e-$ ребро этого подграфа. По ребру $e$ можно найти компоненту связности $W_{e}$ графа $\Delta_{G}$ не более, чем за $C^{\prime} \cdot n^{4}$ операций, поскольку порядок графа $\Delta_{G}$ не превосходит $n^{2}$, а трудоемкость нахождения всех компонент связности графа порядка $p$ не превосходит величины, пропорциональной $p^{2}$ (см. [8]).

Далее рассмотрим алгоритм получения замкнутой совокупности компонент связности графа $\Delta_{G}$, содержащей компоненту $W_{e}$.

1) Положим $s=1$ и в качестве совокупности $W_{1}, \ldots, W_{s}$ выберем одну компоненту $W_{e}$. 
2) Строим множество $M\left(W_{1}, \ldots, W_{s}\right) \subset V(G)$.

3) Для каждой пары различных вершин $a, b$ из $M\left(W_{1}, \ldots, W_{s}\right)$ проверяем, лежит ли ребро $(a, b)$ в одной из компонент $W_{1}, \ldots, W_{s}$.

4) Если в п.3 проверяемое условие выполняется для всех пар, то $W_{1}, \ldots, W_{s}$ является замкнутой совокупностью и в графе $G$ есть нетривиальный выделенный подграф - порожденный подграф с множеством вершин $M\left(W_{1}, \ldots, W_{S}\right)$.

5) Если в п.3 обнаруживается такая пара вершин $a, b$ из $M\left(W_{1}, \ldots, W_{s}\right)$, что ребро $(a, b)$ лежит в компоненте связности $W$ и эта компонента не входит в совокупность $W_{1}, \ldots, W_{s}$, то вместо совокупности $W_{1}, \ldots, W_{s}$ рассмотрим совокупность $W_{1}, \ldots, W_{s}, W$ из $s+1$ компонент. Если $W_{1}, \ldots, W_{s}, W-$ все компоненты связности графа $\Delta_{G}$, то в графе $G$ нет нетривиального выделенного подграфа, содержащего ребра из компоненты $W_{e}$. В противном случае полагаем $W_{s+1}=W$, заменяем $s$ на $s+1$ и переходим к п. 2 .

Собственно метод проверки наличия нетривиального выделенного подграфа заключается в том, что предложенный алгоритм необходимо выполнить для каждой компоненты связности графа $\Delta_{G}$ до получения первого успеха. Если ни для одной компоненты $W$ не найдется нетривиального выделенного подграфа графа $G$, содержащего ребра из компоненты $W$, то в графе $G$ вообще нет нетривиальных выделенных подграфов.

Трудоемкость построенного метода оценивается сверху величиной $C^{\prime \prime} \cdot n^{8}$. Действительно, получить множество $M\left(W_{1}, \ldots, W_{s}\right) \subset V(G)$ можно не более, чем за $n^{5}$ операций (для каждой из $n$ вершин графа $G$ проверим, инцидентна ли она одному из ребер, составляющих компоненты $W_{1}, \ldots, W_{s}$, $s<n^{2}$; размер каждой компоненты связности не превосходит $n^{2}$ ). Трудоемкость п. 3 оценивается сверху величиной $n^{6}$ (всего не более $n^{2}$ пар вершин, а проверка вхождения ребра в совокупность $W_{1}, \ldots, W_{s}$ не превосходит суммарного размера совокупности, который, в свою очередь, меньше $n^{4}$ ). Трудоемкости пп. 3-5 незначительны по сравнению с предыдущими пунктами. Значит, алгоритм получения замкнутой совокупности компонент связности графа $\Delta_{G}$, содержащей фиксированную компоненту $W$, имеет трудоемкость не более $C^{\prime \prime} \cdot n^{6}$, и в самом худшем случае этот алгоритм придется выполнить для каждой из компонент графа $\Delta_{G}$, число которых не превышает $n^{2}$.

Сформулируем доказанный результат.

Утверждение 4. Проверить наличие выделенного подграфа в графе $G$ порядка п и, в случае положстельного ответа, найти этот подграф, можно с трудоемкостью, не превышающей $C^{\prime \prime} \cdot n^{8}$, где $C^{\prime \prime}$ - константа. 
Укажем несколько случаев, когда существует очевидная возможность редукции графа в задаче поиска минимального РПК, и покажем, что в этих случаях в графе существует нетривиальный выделенный подграф.

Утверждение 5. Если граф G обладает одним из следующих свойств:

1) граф $G$ не связный;

2) в графе $G$ есть точка сочленения;

3) в графе $G$ есть ребро, которое не лежит ни в одном треугольнике, то в графе $G$ сущчествует нетривиальный выделенный подграф.

Доказательство. Если граф $G$ не связный, то нетривиальным выделенным подграфом является любая компонента связности. Если в графе $G$ есть точка сочленения, то существует блок с хотя бы одним ребром, не совпадающий со всем графом; этот блок и является искомым нетривиальным выделенным подграфом. Если в графе $G$ есть ребро $(a, b)$, которое не лежит ни в одном треугольнике, то выделенным подграфом является подграф, порожденный вершинами $a$ и $b$.

\section{§ 2. Компакты в графе}

Воспользуемся определением собственного ребра подграфа $H$ для случая, когда $H$ есть замкнутое окружение некоторой вершины $x$. Тогда ребро $e$ окружения $N(x)$ собственное, если не существует треугольника со стороной $e$ и вершиной, не лежащей в замкнутом окружении $N^{*}(x)$. Это проиллюстрировано на рис. 1.

Для этого случая лемму 1 можно слегка усилить.

Лемма 5. Если ребро е графа $G$ является собственным ребром замкнутого окружения вершины $x$, то в любом РПК графа $G$ все клики, содержашие ребро е, иеликом лежат в подграфе $N^{*}(x)$ и содержат вершину $x$.

Доказательство. Пусть $K_{1}, \ldots, K_{r}-$ РПК графа $G$ и клика $K_{i}$ содержит указанное в условии ребро $e=(\tau, w)$. По лемме 1 все вершины клики $K_{i}$ лежат в подграфе $N^{*}(x)$. Так как вершина $x$ по определению смежна со всеми вершинами из $N(x)$, то она смежна со всеми вершинами клики $K_{i}$. В силу максимальности клики $K_{i}$ получаем, что $x \in K_{i}$.

Отметим, что подграф $N_{G}(x)$ по определению не содержит вершину $x$ и поэтому целиком лежит в графе $G \backslash\{x\}$ (но в $G \backslash\{x\}$ этот подграф не обязан, вообще говоря, являться окружением какой-то вершины). Следующая лемма показывает, что собственное ребро замкнутого окружения либо инцидентно вершине $x$, либо является собственным ребром окружения. 


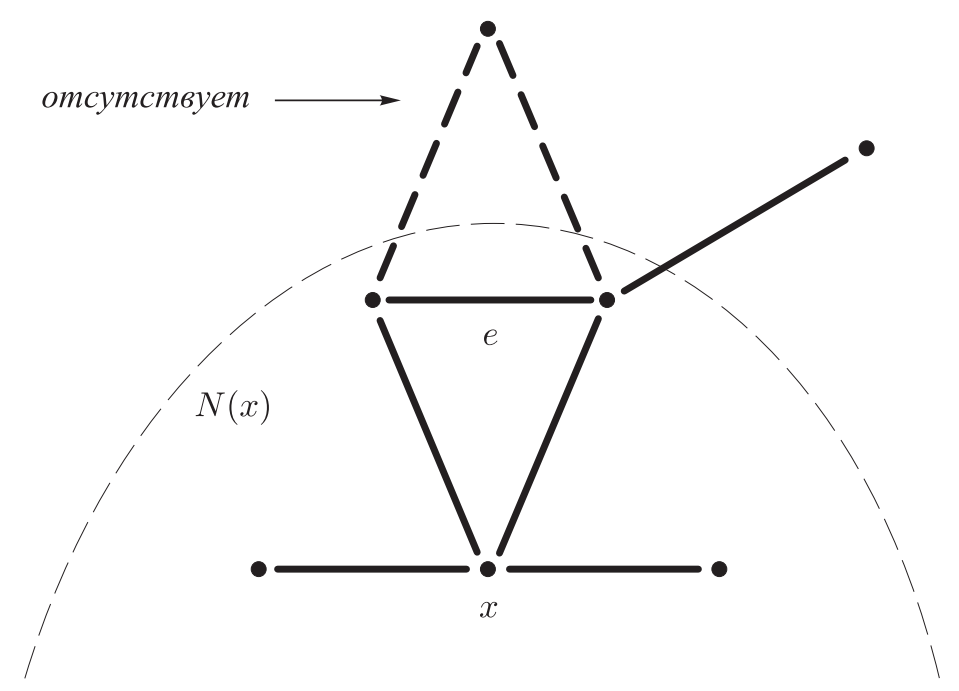

Рис. 1.

Лемма 6. Ребро е, не инцидентное вершине $x$, в графе $G$ является собственным ребром замкнутого окружения $N_{G}^{*}(x)$ тогда и только тогда, когда в графе $G \backslash\{x\}$ ребро е является собственным ребром подграфа $N_{G}(x)$.

Доказательство. Пусть ребро $e=(v, w)$ графа $G$ - собственное ребро замкнутого окружения $N_{G}^{*}(x)$. По определению это эквивалентно условию: если $\{v, w, z\}$ - треугольник в $G$, то вершина $z$ либо лежит в $N_{G}(x)$, либо совпадает с вершиной $x$. Но это происходит тогда и только тогда, когда в графе $G \backslash\{x\}$ для любого треугольника $\{v, w, z\}$ вершина $z$ лежит в $N_{G}(x)$. Последнее условие эквивалентно тому, что в графе $G \backslash\{x\}$ ребро $e$ является собственным ребром подграфа $N_{G}(x)$.

Введем еще одно определение. Окружение вершины $x$ в графе $G$ назовем компактом, если множество собственных ребер замкнутого окружения $N^{*}(x)$, не инцидентных вершине $x$, образует реберное покрытие вершин подграфа $N(x)$. Другими словами, $N(x)$ является компактом тогда и только тогда, когда для каждой вершины $y$ окружения $N(x)$ найдется такая другая вершина $z$ окружения $N(x)$, что ребро $(y, z)$ является собственным ребром замкнутого окружения $N^{*}(x)$.

Неформально говоря, компакт - это окружение вершины, содержащее в некотором смысле много собственных ребер. Отметим, что, согласно определению, окружение изолированной вершины $x$ (пустое множество) также является компактом. 
Следующее утверждение показывает, что наличие в графе компакта позволяет упрощать задачу поиска минимального РПК.

Утверждение 6. Пусть в графе $G$ существует непустое окружение вершины $x$, являюшееся компактом. Тогда произвольное РПК $\Psi$ графа $G \backslash\{x\}$ дает РПК графа $G$, если добавить вершину х в те клики из $\Psi$, которые содержат собственные ребра компакта $N(x)$. Применение к всевозможным РПК $\Psi$ графа $G \backslash\{x\}$ указанной прочедуры дает все РПК графа $G$.

Доказательство. Пусть $\Psi$ - произвольное РПК графа $G \backslash\{x\}$. Пусть $U$ - множество всех собственных ребер замкнутого окружения $N^{*}(x)$, не инцидентных $x$, и $K_{1}^{\prime}, \ldots, K_{s}^{\prime}-$ все клики из РПК $\Psi$, каждая из которых содержит хотя бы одно ребро из $U$. Рассмотрим без ограничения общности клику $K_{i}, i \in\{1,2, \ldots, s\}$ и обозначим через $е$ ребро из множества $U$, лежащее в $K_{i}$. По лемме 6 в графе $G \backslash\{x\}$ ребро $e$ является собственным ребром подграфа $N(x)$, следовательно, по лемме 1 клика $K_{i}$ целиком лежит в $N(x)$. Значит, в графе $G$ все вершины клики $K_{i}^{\prime}$ смежны с вершиной $x$, поэтому вершину $x$ можно добавить в клику $K_{i}^{\prime}$, получив тем самым клику $K_{i}$ графа $G$. Поскольку клика $K_{i}^{\prime}$ - максимальная в графе $G \backslash\{x\}$, постольку клика $K_{i}$ оказывается максимальной в графе $G$. Заменим в наборе $\Psi$ каждую из клик $K_{i}^{\prime}$ кликой $K_{i}$. Полученный набор обозначим через $\Omega$ и докажем, что $\Omega$ есть РПК графа $G$. Действительно, все ребра графа $G$, принадлежащие подграфу $G \backslash\{x\}$, очевидно, покрываются кликами набора $\Omega$. Осталось рассмотреть только ребра вида $(x, y)$, где $y-$ вершина из $N(x)$. Так как $N(x)$ - компакт, то по определению для любой вершины $y$ найдется ребро в $U$, инцидентное $y$. Следовательно, в наборе $\Psi$ найдется клика $K_{j}^{\prime}, j \in\{1,2, \ldots, s\}$, которая содержит это ребро (и, соответственно, вершину $y$ ). В наборе $\Omega$ клика $K_{j}^{\prime}$ заменена кликой $K_{j}=K_{j}^{\prime} \cup\{x\}$, которая содержит ребро $(x, y)$, т. е. все такие ребра покрываются набором клик $\Omega$. Значит, $\Omega-$ РПК графа $G$.

Теперь докажем, что любое РПК $\Omega$ графа $G$ можно получить из какогото РПК графа $G \backslash\{x\}$ процедурой, описанной в формулировке утверждения. Пусть снова $U$ - все собственные ребра замкнутого окружения $N^{*}(x)$, не инцидентные вершине $x$; клики $K_{1}, \ldots, K_{s}-$ все клики из РПК $\Omega$, каждая из которых содержит хотя бы одно ребро из $U$. Тогда по лемме 5 каждая из клик $K_{i}$, $i \in\{1,2, \ldots, s\}$, целиком содержится в подграфе $N^{*}(x)$ и содержит вершину $x$. Удалим из каждой клики $K_{i}$ вершину $x$ : для каждого $i \in\{1,2, \ldots, s\}$ рассмотрим клику $K_{i}^{\prime}=K_{i} \backslash\{x\}$. Рассмотрим набор клик $\Psi$, полученный из набора $\Omega$ заменой каждой из клик $K_{i}$ на $K_{i}^{\prime}$. Так как этот набор покрывает все ребра графа $G$ за исключением ребер, инцидентных вершине $x$, то $\Psi$ является РПК графа $G \backslash\{x\}$. Кроме того, клики $K_{i}^{\prime}$ максимальны в графе $G \backslash\{x\}$ : 
в противном случае клики $K_{i}$ не максимальны в $G$. Значит $\Psi-$ РПК графа $G$, причем покрытие именно максимальными кликами. Очевидно по построению, что набор клик $\Omega$ получается из набора клик $\Psi$ процедурой добавления вершины $x$ в клики $K_{i}^{\prime}, i \in\{1,2, \ldots, s\}$, т. е. во все клики, которые содержат хотя бы одно ребро из множества $U$.

Отметим, что если в графе $G$ существует изолированная вершина $x$, то ее окружение (пустое множество) является компактом, и тогда утверждение по сути остается верным, только процедура получения $\Omega$ из $\Psi$ выглядит иначе: просто надо к набору максимальных клик $\Psi$ добавить клику, состоящую из одной вершины $x$. Таким образом, наличие в графе компакта во всех случаях (в том числе и в вырожденном) означает возможность редукции графа.

Следствие 3. В условиях утверждения 6 верно равенство $\Theta(G)=$ $=\Theta(G \backslash\{x\})$.

Доказательство. Для любого порожденного подграфа $H$ графа $G$ верно неравенство $\Theta(H) \leq \Theta(G)$. Действительно, возьмем произвольное РПК $\Omega$ графа $G$ из $r=\Theta(G)$ максимальных клик и оставим в каждой из клик набора $\Omega$ только те вершины, которые лежат в подграфе $H$. Поскольку $H-$ порожденный подграф, такая процедура приведет к набору $\Psi$ именно клик (не обязательно максимальных; некоторые из клик могут оказаться пустыми). Дополним каждую из клик набора $\Psi$ произвольным образом до максимальной. Тогда набор $\Psi$ будет РПК подграфа $H$ и число клик в наборе $\Psi$ будет не более $r$. Значит $\Theta(H) \leq r=\Theta(G)$.

Применив полученное неравенство к случаю, когда $H$ есть подграф $G \backslash\{x\}$ (очевидно, это порожденный подграф), получим: $\Theta(G \backslash\{x\}) \leq \Theta(G)$.

С другой стороны, если непустое окружение вершины $x$ является компактом, то по утверждению 6 РПК графа $G \backslash\{x\}$ из $\Theta(G \backslash\{x\})$ клик можно добавлением в некоторые клики вершины $x$ превратить в РПК графа $G$ из такого же числа клик. Следовательно, существует РПК графа $G$ из $\Theta(G \backslash\{x\})$ клик, и поэтому $\Theta(G \backslash\{x\}) \geq \Theta(G)$.

Объединяя два неравенства, получаем утверждение следствия.

Если окружение вершины не является компактом, то утверждение 6 и следствие из него, вообще говоря, не верны. Приведем пример. Рассмотрим граф $G$ слева на рис. 2.

У графа $G$ существует единственное РПК: $\left\{x, a_{4}\right\},\left\{x, a_{1}, a_{2}\right\}$, $\left\{x, a_{2}, a_{3}\right\},\left\{a_{1}, a_{2}, b_{1}\right\},\left\{a_{2}, a_{3}, b_{2}\right\}$; поэтому $\Theta(G)=5$. Однако для графа $G \backslash\{x\}$ (справа на рис. 2) тоже существует единственное РПК: $\left\{a_{4}\right\}$, $\left\{a_{1}, a_{2}, b_{1}\right\},\left\{a_{2}, a_{3}, b_{2}\right\} ; \Theta(G \backslash\{x\})=3 \neq \Theta(G)$. 

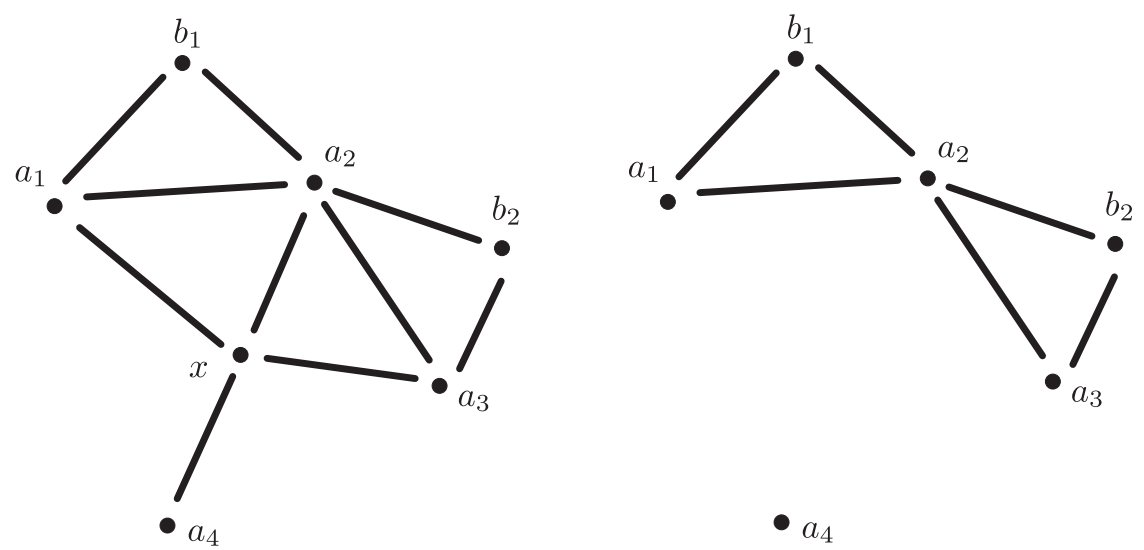

Рис. 2.

Из утверждения 6 вытекает следующий алгоритм поиска минимального РПК в графе $G$. Сначала надо найти такую вершину $x$ в графе $G$, что ее окружение является компактом; затем удалить вершину $x$ (и инцидентные ей ребра), перейдя к поиску минимального РПК для графа $G \backslash\{x\}$. Причем для графа $G \backslash\{x\}$ можно снова искать компакты и редуцировать граф. Затем, найдя РПК графа $G \backslash\{x\}$ минимальным числом клик, добавим вершину $x$ в клики этого РПК, содержащие собственные ребра компакта $N(x)$, и получим граф $G$.

Рассмотрим вопрос о трудоемкости поиска компакта в графе $G$. Предложим следующий алгоритм поиска компакта (см. рис. 3).

1. Перебираем все вершины $x$ графа $G$. Для каждой вершины $x$ проверяем, является ли окружение $N(x)$ компактом. Для этого переходим к п. 2.

2. Перебираем все смежные с $x$ вершины $y$. Проверяем, для каждой ли вершины $y$ из $N(x)$ найдется инцидентное ей собственное ребро замкнутого окружения $N^{*}(x)$, не инцидентное вершине $x$. Для этого переходим к п. 3.

3. Перебираем все вершины $z$, смежные и с $x$, и с $y$, т. е. перебираем все ребра окружения, инцидентные вершине $y$. Для того, чтобы проверить, что ребро $(y, z)$ - собственное ребро $N(x)$, переходим к п. 4.

4. Перебираем все вершины $v$, смежные с $y$. Если найдется такая вершина $v$, что $v$ смежна с $z$ и не смежна с $x$, то ребро $(y, z)$ не собственное; если такой вершины не найдется, то ребро $(y, z)$ собственное. 


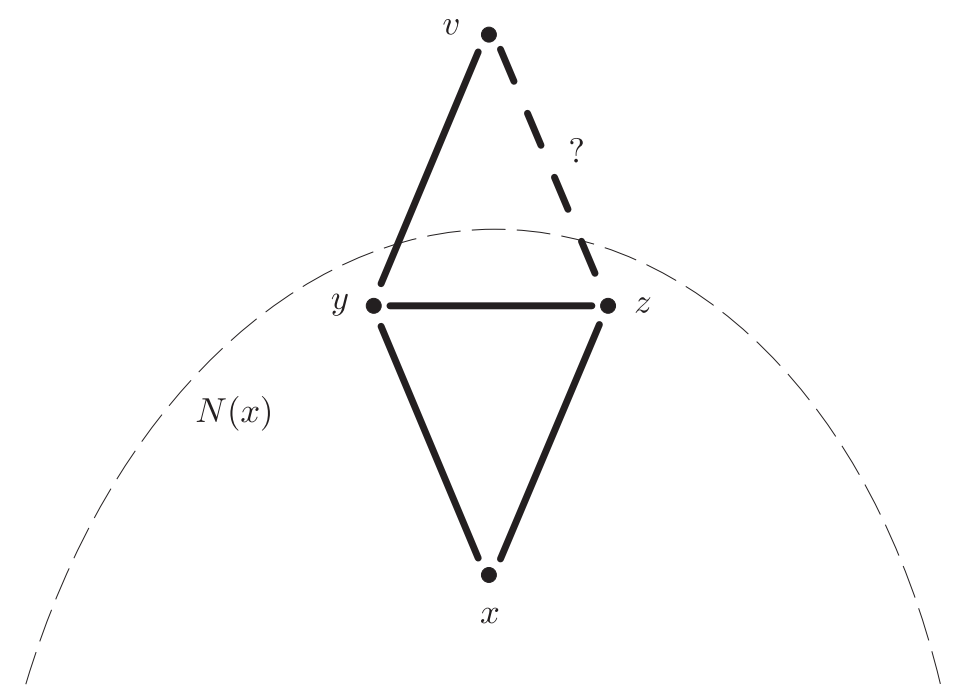

Рис. 3.

Трудоемкость построенного алгоритма оценим в том же предположении, что и в § 1: считаем, что граф хранится в виде матрицы смежности, и поэтому существование ребра устанавливается за одно обращение в память. Тогда перебрать все вершины, смежные с фиксированной, можно за $n$ операций. Следовательно, трудоемкость построенного алгоритма оценивается величиной $C \cdot n^{4}$, где $C$ - некоторая константа. Таким образом, доказано следующее утверждение.

Утверждение 7. Все компакты графа $G$ порядка $n$ можно найти с трудоемкостью, не превымающей $C \cdot n^{4}$, где $C$ - константа, не зависящая om $n$.

\section{§3. Частные случаи компакта}

Объекты, приводящие к редукции графа, т. е. вершины, ребра или окружения вершин с определенными свойствами, уже вводились ранее. Отдельно стоит упомянуть сравнительно недавнюю работу [3], в которой приведен наиболее полный список таких объектов, в том числе введен один новый объект. В этом параграфе мы покажем, что все эти объекты являются относительно простыми частными случаями компакта.

Первым из таких объектов является доминирующая вершина в графе. Доминирующей вершиной в графе называется вершина, смежная со всеми остальными вершинами графа. 
Утверждение 8. Если в графе $G$ существует доминирующая вершина $x$ и граф $G \backslash\{x\}$ связен, то в графе $G$ есть компакт.

Доказательство. Пусть $x$ - доминирующая вершина в графе $G$. Рассмотрим замкнутое окружение $N^{*}(x)$. Оно совпадает со всем графом $G$. Любое ребро этого графа будет, очевидно, собственным ребром его же самого. Поскольку граф $G \backslash\{x\}$ связен, множество всех ребер графа $G \backslash\{x\}$ образует его реберное покрытие вершин.

Рассмотрим объект, введенный в работе [3], сохранив предложенную авторами терминологию. Пусть $x$ - вершина графа $G$ и $y$ - вершина из окружения $N(x)$. Вершину у назовем «заключенной» («prisoner»), если любая вершина $z$, смежная с $y$ в графе $G$, лежит в $N^{*}(x)$, и «выходящей» («exit») в противном случае. Скажем, что «заключенные» доминируют над «выходящими», если для каждой вершины $v$ из окружения $N(x)$ найдется вершина«заключенная», смежная с $v$. Подходящим для редукции задачи поиска минимального РПК является граф, имеющий вершину, в окружении которой «заключенные» доминируют над «выходящими».

Утверждение 9. Если в графе $G$ существует окружение вершины х, в котором «заключенные» доминируют над «выходящими», то это окружение является компактом.

Доказательство. Для каждой вершины $y$ из подграфа $N(x)$ существует вершина-«заключенная» $z$, смежная с $y$. Поскольку из вершины $z$ не выходят ребра, соединяющие ее с вершинами графа $G$, лежащими вне подграфа $N^{*}(x)$, то в графе $G$ не может существовать таких треугольников $\{y, z, v\}$, что $v$ не лежит в $N^{*}(x)$. Следовательно, ребро $(y, z)$ является собственным ребром замкнутого окружения $N^{*}(x)$, причем не инцидентным вершине $x$. Таким образом, множество собственных ребер замкнутого окружения $N^{*}(x)$, не инцидентных вершине $x$, образует реберное покрытие вершин подграфа $N(x)$. Следовательно, $N(x)-$ компакт.

Отметим, что понятие компакта является более широким, чем доминирование «заключенных» над «выходящими»; например для графа $G$, приведенного на рис. 4, окружение вершины $x$ является компактом, однако в этом окружении вообще нет вершин-«заключенных», и поэтому в $N(x)$ «заключенные» не доминируют над «выходящими».

Две вершины $x, y$ графа $G$ называются эквивалентными, если $N^{*}(x)=$ $=N^{*}(y)$. Легко видеть, что введенное отношение действительно является отношением эквивалентности на множестве вершин графа $G$, т. е. $V(G)$ разбивается на множество классов эквивалентности. Понятие эквивалентных 


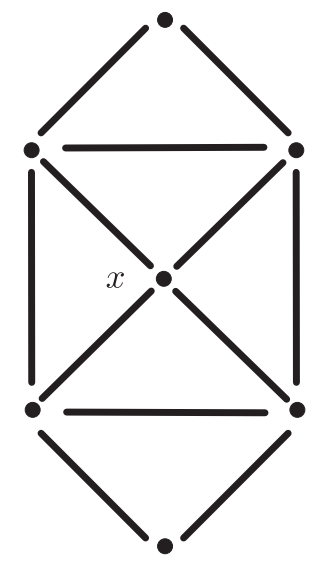

Рис. 4

вершин использовано для редукции графа одним из первых. Отметим, что оно достаточно содержательно и само по себе; в частности, зафиксировав максимальную мощность класса эквивалентности, можно получить оценку, связывающую кликовое число графа $\Theta(G)$ и его порядок $n=|V(G)|$. Для этого сначала укажем связь между РПК графа и классами эквивалентности его вершин.

Утверждение 10. Пусть $K_{1}, \ldots, K_{r}-$ РПК графа $G$, в котором все клики различны; с каждым подмножеством $I \subseteq\{1, \ldots, r\}, I \neq \varnothing$, свяжем множество вершин графа $M_{I}=\left(\bigcap_{i \in I} K_{i}\right) \backslash\left(\bigcup_{\substack{j=1 \\ j \notin I ;}}^{r} K_{j}\right)$. Тогда каждое множе-

ство $M_{I}$ либо пусто, либо совпадает с некоторым классом эквивалентности вершин графа $G$. Кроме того, множества $M_{I}$ и $M_{J}$ не пересекаются при различных I и J, объединение $\bigcup \quad M_{I}$ исчерпывает все множество вериин графа $G$.

Доказательство. Пусть $I$ - произвольное подмножество в $\{1, \ldots, r\}$. Докажем, что любая вершина $a$ из множества $M_{I}$ смежна со всеми вершинами максимальных клик $K_{i}, i \in I$, отличными от $a$, и только с ними. Действительно, пусть для некоторого $i \in I$ вершина $b$ лежит в максимальной клике $K_{i}$. Тогда вершины $a$ и $b$ смежны, поскольку по определению множества $M_{I}$ вершина $a$ обязана, в частности, лежать в клике $K_{i}$. Пусть теперь вершина $b$ смежна с вершиной $a$. Найдется максимальная клика $K_{i}$, содержащая ребро 
$(a, b)$ (возможно не одна; тогда возьмем любую из них). В частности, клика $K_{i}$ содержит вершину $a$; поэтому $i \in I$. Действительно, если $i$ не принадлежит $I$, то а принадлежит множеству $\left(\bigcup_{j \in R \backslash I} K_{j}\right)$ и, следовательно, не может лежать в множестве $M_{I}$.

Из доказанного следует, что у всех вершин из одного и того же множества $M_{I}$ их замкнутые окружения совпадают и равны $\bigcup_{i \in I} K_{i}$. Следовательно, в $M_{I}$ все вершины эквивалентны. Поскольку по условию все клики $K_{1}, \ldots, K_{r}$ различны, то замкнутые окружения вершин из множества $M_{J}$, равные $\bigcup_{i \in J} K_{i}$, при $I \neq J$ не совпадают с $\bigcup_{i \in I} K_{i}$. Следовательно, $M_{I} \cap M_{J}=\varnothing$ при $I \neq J$.

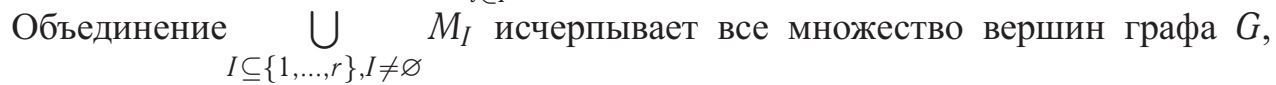
поскольку для каждой вершины $a$ графа $G$ однозначно можно указать все те клики $K_{i_{1}}, \ldots, K_{i_{p}}$ из набора $K_{1}, \ldots, K_{r}$, которые содержат вершину $a$. Тогда очевидно:

$$
a \in M_{\left\{i_{1}, \ldots, i_{p}\right\}}=\left(K_{i_{1}} \cap \ldots \cap K_{i_{p}}\right) \backslash\left(\bigcup_{j \in\{1, \ldots, r\} \backslash\left\{i_{1}, \ldots, i_{p}\right\}} K_{j}\right) .
$$

Таким образом, в $M_{I}$ все вершины эквивалентны; всякая вершина графа $G$, не принадлежащая $M_{I}$, лежит в каком-то $M_{J}, I \neq J$, поэтому имеет другое замкнутое окружение и не эквивалентна вершинам из $M_{I}$. То есть $M_{I}$ либо пусто, либо является классом эквивалентности.

Доказанное утверждение можно переформулировать в следующем виде: если из совокупности $\left\{M_{I}: I \subseteq\{1, \ldots, r\}, I \neq \varnothing\right\}$ удалить те из множеств $M_{I}$, которые пусты, то полученная совокупность будет представлять собой разбиение множества вершин графа $G$ на классы эквивалентности. Из этого можно сделать несколько следствий.

Следствие 4. Если порядок графа $G$ равен $n, K_{1}, \ldots, K_{r}-P \Pi K$ графа $G$ u множество вершин графа $G$ разбивается на s классов эквивалентности, mo $s \leq 2^{r}-1$.

Доказательство. Всего в совокупности $\left\{M_{I}: I \subseteq\{1, \ldots, r\}, I \neq \varnothing\right\}$ имеется $2^{r}-1$ множеств $M_{I}$. Каждое из них, которое не пусто, совпадает с некоторым классом эквивалентности вершин графа $G$. Так как предыдущему утверждению $M_{I} \cap M_{J}=\varnothing$ при $I \neq J$, то различные непустые $M_{I}$ и $M_{J}$ совпадают с различными классами. Следовательно, получаем оценку $s \leq 2^{r}-1$. 
Следствие 5. Если в условиях следствия 4 мощность наибольшего класса эквивалентности равна $t(G) \geq 1$, то

$$
\Theta(G) \geq \log _{2}\left(\frac{n}{t(G)}+1\right) .
$$

Доказательство. Всего классов эквивалентности $s$, и каждый из этих классов содержит не более $t(G)$ вершин. Следовательно, s.t $(G) \geq n$. Рассмотрев РПК графа $G$ из $\Theta(G)$ клик и воспользовавшись оценкой из предыдущего следствия, получаем, что $\left(2^{\Theta(G)}-1\right) \cdot t(G) \geq n$. Отсюда получаем требуемое неравенство.

Отметим, что если в графе $G$ нет эквивалентных вершин, то $t(G)=1$ и следствие 5 дает известную оценку для такого класса графов: $\Theta(G) \geq$ $\geq \log _{2}(n+1)$ (см. работу [6]).

Теперь докажем, что эквивалентные вершины являются частным случаем компакта в графе.

Утверждение 11. Если в графе $G$ существует две эквивалентные вериины, то в графе $G$ есть компакт.

Доказательство. Если две вершины $x$ и $y$ эквивалентны, то в окружении $N(x)$ вершины $x$ вершина $y$ является доминирующей, поскольку она смежна с любой вершиной, с которой смежна вершина $x$ (за исключением самой $y$ ). Вместе с тем не может существовать треугольника $\{y, z, v\}$, где вершина $z$ лежит в $N(x)$ и вершина $v$ не лежит в $N(x)$, поскольку тогда $y$ смежна с $v$, а $x$ не смежна с $v$, что противоречит эквивалентности вершин $x$ и $y$. Таким образом, множество ребер $(y, z)$, где вершина $z$ - произвольная вершина $N(x)$, отличная от $y$, образует реберное покрытие вершин графа $N(x)$ собственными ребрами. То есть $N(x)$ - компакт.

\section{§4. Собственные ребра клик}

В этом параграфе рассмотрим собственные ребра клик. Формально здесь не предложен способ использования таких ребер для редукции графа в задаче поиска минимального РПК. Однако такие ребра обладают рядом интересных свойств, позволяющих заранее указывать некоторые клики в составе минимального РПК или, в частных случаях, вообще получать единственно возможное РПК графа. Верна следующая лемма, дающая эквивалентное определение собственного ребра клики. 
Лемма 7. Ребро е является собственным ребром клики $K$ тогда и только тогда, когда оно лежит в этой клике и не лежит ни в какой другой клике графа $G$.

Доказательство. Для доказательства заметим, что существование треугольника, одной стороной из которого является ребро $e$, а третья вершина не лежит в клике $K$, очевидно эквивалентно существованию клики, целиком не лежащей в клике $K$ и содержащей ребро $e$.

Клику $K$, имеющую хотя бы одно собственное ребро, назовем зафиксированной. Такое название продиктовано следующим простым утверждением.

Утверждение 12. Клика $K$ входит в любое РПК графа $G$ тогда и только тогда, когда клика К является зафиксированной.

Доказательство. Пусть клика $K$ является зафиксированной, т.е. содержит ребро $e$, являющееся собственным. Поскольку любое РПК состоит из клик, которые покрывают в совокупности все ребра графа, постольку в каждом РПК присутствует как минимум одна клика, покрывающая ребро $e$, а поскольку такая клика единственна в графе (клика $k$ ), клика $K$ входит в любое РПК графа $G$.

Пусть теперь клика $K$ не зафиксированная. Тогда для каждого ребра $e$ клики $K$ существует клика $K_{e}$, отличная от $K$, которая содержит ребро $e$. Пусть для графа $G$ существует РПК $P$, содержащее клику $K$. Удалим из набора $P$ клику $K$ и для каждого ребра $e$ из клики $K$ добавим в набор клику $K_{e}$. Очевидно, что полученный набор клик $P^{\prime}$ является РПК графа $G$, не содержащим клику $K$. Следовательно, клика $K$ в этом случае не входит во все РПК графа $G$.

Один важный частный случай описывается очевидным следствием из доказанного утверждения.

Следствие 6. Если множество зафиксированных клик образует РПК графа, то это будет РПК минимальным числом клик.

Собственные ребра и, соответственно, зафиксированные клики, допускают простую характеризацию, позволяющую легко распознать их в графе.

Утверждение 13. Ребро е графа G является собственным ребром клики $K$ тогда и только тогда, когда множество вершин графа $G$, смежных одновременно с обоими кониами ребра е, порождают полный подграф в $G$, причем этот полный подграф в объединении с концами ребра е и образует клику K. 
Доказательство. Пусть ребро $e \in E(G)$ соединяет вершины $a$ и $b$. Предположим сначала, что ребро $e$ является собственным ребром некоторой клики. От противного - пусть в графе существуют несмежные между собой вершины $x$ и $y$, смежные одновременно с $a$ и $b$. Тогда в графе имеются два различных треугольника - $\{a, b, x\}$ и $\{a, b, y\}$. Каждый из этих треугольников дополняется до максимальных клик, но эти клики различны, поскольку вершины $x$ и $y$ несмежны. То есть ребро $e$ лежит более чем в одной клике, что приводит нас к противоречию.

В обратную сторону - предположим существование двух различных максимальных клик $K_{1}$ и $K_{2}$, содержащих ребро $e$. Тогда из их максимальности следует, что должны существовать две несмежные вершины $x \in K_{1}$ и $y \in K_{2}$. Каждая из вершин $x, y$ одновременно смежна с концами ребра $e$, значит, подграф, порожденный $x, y$ и концами ребра $e$, не является полным.

Из доказанного утверждения следует полиномиальная распознаваемость всех зафиксированных клик графа (трудоемкость не более $O\left(n^{4}\right)$, где $n=|V(G)|$, при условии задания графа матрицей смежности).

Отметим, что существование зафиксированной клики никак не связано с существованием компакта в графе, т.е. существование одного не обязательно влечет за собой существование другого.

\section{§5. Расшепление вершин с несвязным окружением}

Используем для упрощения поиска минимального РПК следующую идею. Пусть в графе $G$ есть вершина $x$, окружение которой не является связным графом, то есть $N(x)=N_{1} \cup N_{2}$ - разложение графа $N(x)$ на два непересекающихся по вершинам непустых подграфа. Тогда проведем следующую процедуру. Удалим из графа $G$ вершину $x$ (и все инцидентные ей ребра); затем добавим в граф две вершины $x^{[1]}$ и $x^{[2]}$, соединив ребрами вершину $x^{[1]}$ со всеми вершинами подграфа $N_{1}$ и вершину $x^{[2]}$ со всеми вершинами подграфа $N_{2}$. Полученный граф обозначим как $G[x]$. В графе $G[x]$ на одну вершину больше; число ребер в графе $G$ и графе $G[x]$ совпадает.

Отметим, что $N_{1}$ и $N_{2}$ не обязаны, вообще говоря, являться компонентами связности: например, если $N(x)$ имеет четыре компоненты связности $N^{(1)}, N^{(2)}, N^{(3)}, N^{(4)}$, то в качестве $N_{1}$ можно взять подграф $N^{(1)} \cup N^{(2)}$, а в качестве $N_{2}-N^{(3)} \cup N^{(4)}$.

Несмотря на то, что в графе $G[x]$ больше вершин, задача поиска минимального РПК для этого графа имеет такую же сложность, что и для графа $G$, поскольку верно следующее утверждение. 
Утверждение 14. Пусть в графе $G$ окружение вершины $x$ не связно, то есть $N(x)=N_{1} \cup N_{2}$ - разложение графа $N(x)$ на два непересекающихся по вершинам непустых подграфа. Поставим в соответствие произвольному РПК $\Psi$ графа $G[x]$ набор клик $\Omega(\Psi)$ графа $G$ следуюшим образом: заменим каждое вхождение любой из вершин $x^{[1]}$ и $x^{[2]}$ в клики РПК $\Psi$ вершиной $x$. Тогда набор клик $\Omega(\Psi)$ является РПК графа $G$ и указанное соответствие есть биекиия множества всех РПК графа $G[x]$ без повторяющихся клик на множество всех РПК графа $G$ без повторяющихся клик.

Доказательство. Сначала докажем, что набор клик $\Omega(\Psi)$ является РПК графа $G$. Очевидно, что клики набора $\Omega(\Psi)$ являются максимальными. Все ребра графа $G$, не инцидентные вершине $x$, есть и в графе $G[x]$, поэтому такие ребра покрываются кликами из набора $\Omega(\Psi)$. Рассмотрим произвольное ребро вида $(x, y)$. Вершина $y$ лежит в одном из графов $N_{j}, j=1,2$. Тогда в графе $G[x]$ есть ребро $\left(x^{[j]}, y\right)$, которое покрывается некоторой кликой $K$ из набора $\Psi$. После замены в клике $K$ вершины $x^{[j]}$ вершиной $x$, получится клика $K^{\prime}$ из набора $\Omega(\Psi)$, содержащая ребро $(x, y)$. Следовательно, набор клик $\Omega(\Psi)$ покрывает все ребра графа $G$, то есть является РПК графа $G$.

Докажем теперь, что введенное соответствие (функция, переводящая РПК графа $G[x]$ в РПК графа $G$; обозначим ее через $F$ ) является биекцией. Докажем сначала инъективность функции $F$. Пусть $\Omega_{1}$ и $\Omega_{2}-$ произвольные РПК графа $G[x]$, в которых нет повторяющихся клик. Тогда, если функция $F$ переводит их в одно и то же РПК графа $G$, то различаться эти РПК могут только в кликах, содержащих вершины $x^{[1]}$ или $x^{[2]}$, поскольку другие клики замена вершин $x^{[j]}, j=1,2$ на вершину $x$ не затрагивает. Пусть теперь $K_{1}, \ldots, K_{s}-$ клики РПК $\Omega_{1}$, содержащие вершины $x^{[1]}$ или $x^{[2]}$, и $L_{1}, \ldots, L_{t}-$ клики РПК $\Omega_{2}$, содержащие вершины $x^{[1]}$ или $x^{[2]}$. Отметим, что вершины $x^{[1]}$ и $x^{[2]}$ несмежны, поэтому никакая клика графа $G[x]$ не содержит их одновременно. Более того, по построению $N_{G[x]}\left(x^{[1]}\right) \cap N_{G[x]}\left(x^{[2]}\right)=\varnothing$, поэтому нет пары клик в графе $G[x]$, одна из которых содержит вершину $x^{[1]}$ и произвольную вершину $y$, отличную от $x^{[1]}$ и $x^{[2]}$, а вторая содержит вершину $x^{[2]}$ и вершину $y$. Далее рассмотрим произвольную клику $K_{i}$ из РПК $\Omega_{1}, i \in\{1, \ldots, s\}$. Предположим, что она содержит $x^{[1]}$ (для случая $x^{[2]}$ доказательство аналогично). Тогда функция $F$, заменяя в клике $K_{i}$ вершину $x^{[1]}$ вершиной $x$, создаст некоторую клику $T$ из РПК $F\left(\Omega_{1}\right)$. Так как подграфы $N_{1}$ и $N_{2}$ не пусты, то и в клике $K_{i}$, и в клике $T$ содержится как минимум еще одна вершина $y$, лежащая в $N_{1}$ (она не может лежать в $N_{2}$, поскольку смежна с $\left.x^{[1]}\right)$. Так как мы предположили, что $F\left(\Omega_{1}\right)=F\left(\Omega_{2}\right)$, то в клику $T$ функция $F$ переведет некоторую клику $L_{j}, j \in\{1, \ldots, t\}$. Поскольку вершина $y$ лежит в $N_{1}$, то клика $L_{j}$ содержит вершину $x^{[1]}$, а не $x^{[2]}$. 
Таким образом, получаем, что в кликах $K_{i}$ и $L_{j}$ лежат вершины клики $T$, отличные от $x$, и вершина $x^{[1]}$, т. е. клики $K_{i}$ и $L_{j}$ совпадают. Следовательно, доказано, что для любой клики $K_{i}$ найдется клика $L_{i}$, которая совпадает с $K_{i}$, что означает, что набор $\Omega_{1}$ есть поднабор набора $\Omega_{2}$. Повторяя те же рассуждения, поменяв РПК $\Omega_{1}$ и $\Omega_{2}$ местами, получаем, что наборы $\Omega_{1}$ и $\Omega_{2}$ совпадают. Следовательно функция $F$ инъективна.

Для доказательства сюръективности функции $F$ рассмотрим произвольное РПК $\Omega$ графа $G$ без повторяющихся клик. Пусть без ограничения общности $\Omega=\left\{K_{1}, \ldots, K_{s^{\prime}}, K_{s^{\prime}+1}, \ldots, K_{s^{\prime \prime}}, K_{s^{\prime \prime}+1}, \ldots, K_{r}\right\}$, где клики $K_{1}, \ldots, K_{s^{\prime}}$ и только они содержат ребра вида $(x, y)$, где вершина $y$ лежит в подграфе $N_{1}$; клики $K_{s^{\prime}+1}, \ldots, K_{s^{\prime \prime}}$ и только они содержат ребра вида $(x, y)$, где вершина $y$ лежит в подграфе $N_{2}$; клики $K_{s^{\prime \prime}+1}, \ldots, K_{r}$ и только они не содержат вершину $x$. Тогда заменим в каждой клике $K_{i}, i \in\left\{1, \ldots, s^{\prime}\right\}$ вершину $x$ вершиной $x^{[1]}$; в каждой клике $K_{i}, i \in\left\{s^{\prime}+1, \ldots, s^{\prime \prime}\right\}$ вершину $x$ вершиной $x^{[2]} ;$ клики $K_{i}, i \in\left\{s^{\prime \prime}+1, \ldots, r\right\}$ оставим без изменения. Полученные клики будут максимальными в графе $G[x]$ и будут образовывать РПК этого графа. Очевидно, что функция $F$ переведет такое РПК в РПК $\Omega$ графа $G$.

Вообще говоря, хотя расщепление вершины и приводит к графу с тем же количеством ребер, но число вершин увеличивается, поэтому нельзя утверждать, что происходит редукция графа. Однако, учитывая доказанное утверждение, можно сказать, что на такое увеличение порядка графа можно пойти, поскольку в графе могут образоваться новые объекты, например, компакты. Кроме того, сам граф может распасться на несколько компонент связности, что тоже снижает трудоемкость поиска минимального РПК.

\section{Список литературы}

1. Kellerman E. Determination of keyword conflict // IBM Tech. Disclosure Bull. - 1973. - V. 16. № 2. - P. 544-546.

2. Cavers M.S. Clique partitions and coverings of graphs // MSc Thesis. Waterloo, Ontario, Canada. - 2005.

3. Gramm J., Guo J., Huffner F., Niedermeier R. Data reduction, exact, and heuristic algorithms for clique cover // Proc. $8^{\text {th }}$ Workshop on Algor. Eng. and Exper. - SIAM. - 2006. - P. 86-94.

4. Kou L.T., Stockmeyer L.J., Wong C.K. Cliques with regard to keyword conflicts and intersection graphs // Comm. ACM. - 1978. - V. 21. № 2. P. $135-139$.

5. Orlin J. Contentment in graph theory: Covering graphs with cliques // Indag. Math. - 1977. - V. 39. - P. 406-424. 
6. Gyárfás A. A simple lower bound on edge coverings by cliques // Discrete Math. - 1990. - V. 85. - P. 103-104.

7. Гэри М., Джсонсон Д. Вычислительные машины и труднорешаемые задачи / пер. с англ. - М.: Мир, 1979.

8. Кормен Т., Лейзерсон Ч., Ривест Р. Алгоритмы: построение и анализ: пер. с англ. - М.: МЦНМО, 1999.

9. Харари Ф. Теория графов. - М.: Мир, 1973. 